\title{
Increase in corneal epithelial cell size with extended wear soft contact lenses depends on continuous wearing time
}

Department of

Ophthalmology, Tokyo

Dental College, Chiba,

Japan

$\mathrm{K}$ Tsubota

I Toda

Y Yagi

J Shimazaki

\section{Department of}

Ophthalmology, Keio

University School of

Medicine, Tokyo,

Japan

K Tsubota

I Toda

Department of Ophthalmology, Kameda General Hospital, Chiba, Japan $S$ Hata

Department of Ophthalmology, Showa University, Tokyo, Japan

M Sakata

Correspondence to: Kazuo Tsubota, MD, Department of Ophthalmology, Tokyo Dental College, 11-13 Sugano 5 chome, Ichikawashi, Chiba, Japan 272.

Accepted for publication 11 October 1995

Kazuo Tsubota, Seiichiro Hata, Ikuko Toda, Yukiko Yagi, Miki Sakata, Jun Shimazaki

\begin{abstract}
Aim-Although 1 week of continuous wearing time (CWT) is recommended for extended wear soft contact lenses (EWSCL), there have been few studies concerning the appropriateness of this or other CWTs. The current study was undertaken to examine the effect of various CWTs on the cornea by observing the corneal epithelium.

Methods-Specular microscopy was used to evaluate corneal epithelial changes induced by EWSCL in 19 myopic patients (24.0 (SD 6.7) years), divided into four groups: CWT of $0,1,3$, and 6 days.

Results-The initial mean cell area was $628.2(13.4) \mu \mathrm{m}^{2}$. After 6 months this was $629.3(28.6) \mu \mathrm{m}^{2}$ for daily wear (ns), and 660.0 (40.9) $\mu \mathrm{m}^{2}, 727.7 \quad(49.6) \quad \mu \mathrm{m}^{2}$ $(\mathbf{p}<0.01)$, and 815.5 (67.2) $\mu \mathrm{m}^{2}(\mathbf{p}<0.01)$ for CWT of 1,3 , and 6 days, respectively $(\mathbf{p}<0 \cdot 01)$.

Conclusion-These findings indicate that the corneal epithelium changes proportionally with the CWT; the shorter the CWT, the better the epithelial morphology. This suggests that the CWT for EWSCL be minimised, so as to reduce the potential risk of contact lens related problems.
\end{abstract}

(Br f Ophthalmol 1996; 80: 144-147)

It is well known that among contact lenses, the extended wear soft contact lens (EWSCL) variety is most associated with ulcerative ker-

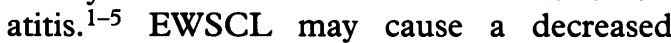
mitotic rate of the corneal epithelium, ${ }^{6}$ decreased corneal sensitivity, ${ }^{7}$ a change in corneal endothelial morphology, 8 and an increased corneal epithelial cell size, as Lemp and we have recently reported..$^{9} 10$

Extended wear contact lenses imply overnight use. Although the longer the continuous wearing time (CWT), the higher the expected complication rate, few studies have analysed the deleterious effects of prolonged CWT. ${ }^{1}$ Since there were many reported cases of ulcerative keratitis with CWT of more than 1 month, the US Food and Drug Administration and its Japanese counterpart (MHW) recommended limiting CWT to 1 week, albeit without a firm scientific basis.

The introduction of disposable soft contact lenses opened a new era for the correction of myopia. Although they have many advantages these lenses still alter the corneal epithelium and pose an increased risk of ulcerative keratitis. $^{210}$ In the frequent replacement programme, patients are instructed to sterilise disposable soft contact lenses every 1 to 4 days, with replacement still recommended after 1 or 2 weeks of use. Whether daily replacement of disposable lenses affords any protection compared with several days of CWT is unknown. Although there are no perfect variables which detect subcellular changes due to contact lens wear, the specular microscopic observation is considered to be a very good method for evaluation of the cellular level. ${ }^{910}$ Specular microscopy can be used for morphometric analysis of the most superficial layer of the cells. The elongation of the cells, as well as their enlargement, is considered to be abnormal at some cellular levels, but this method cannot examine the inner part of the epithelial layers. ${ }^{1112}$ The increase in cell size in the corneal epithelium has been recorded in diabetic cornea, keratoconus, and extended wear soft contact lens wearers as subclinical abnormalities. ${ }^{11-15}$ Since the mean cell size of a normal cornea is maintained around $600 \mu \mathrm{m}^{2}$, the increase in the cell size can be a good indicator for the subclinical evaluation.

In this study, we evaluated the corneal epithelial cell size and shape by specular microscopy in EWSCL users with different CWTs.

\section{Materials and methods}

Thirty seven eyes of 19 myopic patients (10 males, nine females, average age 24.0 (SD 6.7) years) were recruited for this study. They had no ocular surface abnormalities such as allergic conjunctivitis or dry eye. No patients had previously used extended wear contact lenses. The corneal epithelium was normal by slitlamp and specular microscopic observation. SeeQuence (Bausch \& Lomb, Rochester, NY) contact lenses with appropriate refractive power were fitted for each patient. Patients were instructed to remove their lenses daily (group $1, n=6$ ), or every 2 days (group 2, 1 night, $n=5$ ), 4 days (group 3, 2 nights, $n=5$ ), or 7 days (group 4, 6 nights, $n=3$ ). After the assigned CWT, the patients were told to remove their lenses before bedtime, clean them with a thermal disinfection kit (Bausch \& Lomb), and insert the disinfected lenses the next morning. The schedule of lens wear for each group is shown in Figure 1.

The corneal epithelia were photographed through a specular microscope before and 1 week, 1 month, and 6 months after the start of the lens use. Evaluation of the epithelial 
- Removal of contact lens

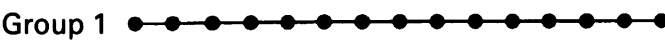

Group 2

Group 3

Group 4

Figure 1 The schedule of extended wear soft contact lens wear in each group. Group $1=$ daily wear, group $2=1$ full day ( 24 hours), group $3=3$ days, group $4=6$ days.

photographs included the number of elongated and large cells which is suggestive of epithelial abnormalities. We also performed morphometric analysis, calculating the mean superficial epithelial cell area.

Furthermore, we compared the mean cell area and the actual CWT in order to see if there was any proportional effect of continuous wear. The effect of an additional day of wear was calculated as follows; the actual increase in cell size divided by the wearing time (days).

\section{Results}

The corneal epithelia were always normal by slit-lamp examination. There were no elongated or large cells before or after the extended wear contact lens use.

The mean superficial corneal epithelial cell areas in each group before wearing the lenses were $643.5(40 \cdot 1) \mu \mathrm{m}^{2}, 611.5(42 \cdot 1) \mu^{2}$ $632.4(67.5) \mu \mathrm{m}^{2}$, and $625.3(69.0) \mu \mathrm{m}^{2}$ respectively, with an overall average of 628.2 (13.4) $\mu \mathrm{m}^{2}$. There were no statistically significant differences among the groups. No changes in cell size were observed at any time in group 1 (Fig 2). However, the mean cell area did increase along with both CWT and the overall duration of lens use. In group 2 , the mean cell area increased to $660 \cdot 0(40 \cdot 9) \mu \mathrm{m}^{2}$ $(\mathrm{p}<0.05)$ at 6 months, whereas no statistically significant differences were found at 1 week or 1 month (Fig 2). In group 3, the mean cell area

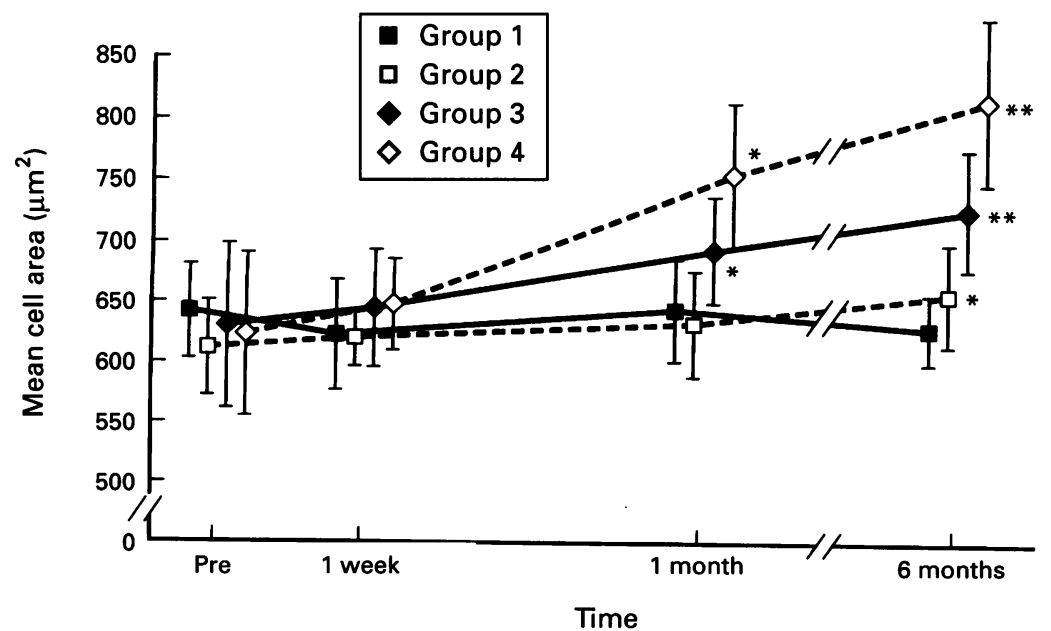

Figure 2 The alterations in corneal epithelial cell size in the four groups. ${ }^{\star} p<0 \cdot 05$; $\star \star p<0 \cdot 01$. increased to $645.6(51.6) \mu \mathrm{m}^{2}(\mathrm{p}>0.05)$ at 1 week, $697 \cdot 2(42 \cdot 7) \mu \mathrm{m}^{2}(\mathrm{p}<0 \cdot 05)$ at 1 month, and $727.7(49 \cdot 6) \mu^{2}$ at 6 months $(p<0 \cdot 01)$. In group 4 , the mean cell area increased to $650.1(37.8) \mu \mathrm{m}^{2}(\mathrm{p}>0.05)$ at 1 week, 754.1 $(58.8) \mu \mathrm{m}^{2}(\mathrm{p}<0.01)$ at 1 month, and 815.5 (67(2) $\mu \mathrm{m}^{2}$ at 6 months ( $<<0.01$ ) (Fig 2). The typical epithelial pictures of each group at 6 months are shown in Figure 3.

Figure 4 shows the relation between the mean cell area and the CWT, which are linearly proportional to each other. Up to 6 days of CWT, the effect of an additional day of wear is to increase the mean cell size by $31.3 \mu \mathrm{m}^{2}$.

\section{Discussion}

EWSCLs were first introduced in 1970 when oxygen permeable soft contact lenses were developed. It was believed that the cornea can function properly as long as the oxygen supply is maintained. ${ }^{16}$ Initially, 1 month of extended wear was accepted, but after many reports of corneal ulcers related to EWSCL the FDA recommended a maximal CWT of 1 week. One study has reported that the longer the CWT, the higher the complication rate. ${ }^{4}$ Even with a CWT of only 1 week, there are still increased relative risks in wearing EWSCL. Perhaps even shorter CWTs would be advisable, although addressing this issue requires choice of an appropriate indicator to follow.

Recent reports indicate that EWSCLs but not other lenses, cause increased cell size in the superficial corneal epithelium. ${ }^{913}$

In this study, we used specular microscopy and morphometric analysis to show that there is linear correlation between CWT of EWSCL and increased corneal epithelial cell size. Increased corneal epithelial cell size is considered to be longer in superficial cells before sloughing off, which may suggest the altered mitosis maturation process of the corneal epithelium. Since the healthy corneal epithelium without infection can be maintained by a proper mitosis maturation process, increased epithelium can be an indicator for the subclinical abnormalities of the corneal epithelium. For up to 1 week of CWT there is an increase in cell size of $31.3 \mu \mathrm{m}^{2}$ per day; although we did not evaluate the corneal epithelium for longer CWT, further increases may be expected up to a point.

Disposable contact lenses were developed to decrease the complication rate with the view of frequent non-compliance with lens hygiene. It is recommended that these lenses be sterilised every 1-7 days and discarded every 1-4 weeks in the frequent replacement programme. From the perspective of corneal epithelial morphology, these lenses should probably be removed and sterilised, if not discarded, every day. Increased cell size may increase epithelial susceptibility to infection and trauma, so that even overnight use of lenses may carry a potential risk. ${ }^{11} 12141517$ This study suggests that the shorter the CWT of soft lenses, the less the chance of corneal complications.

The mechanism of the increased cell size in CWT use is unknown. It is not related to the 


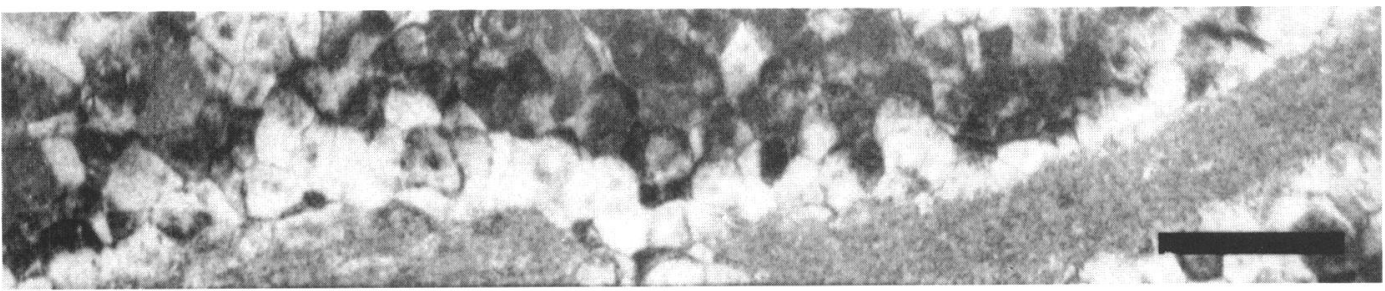

Fig $3 A$

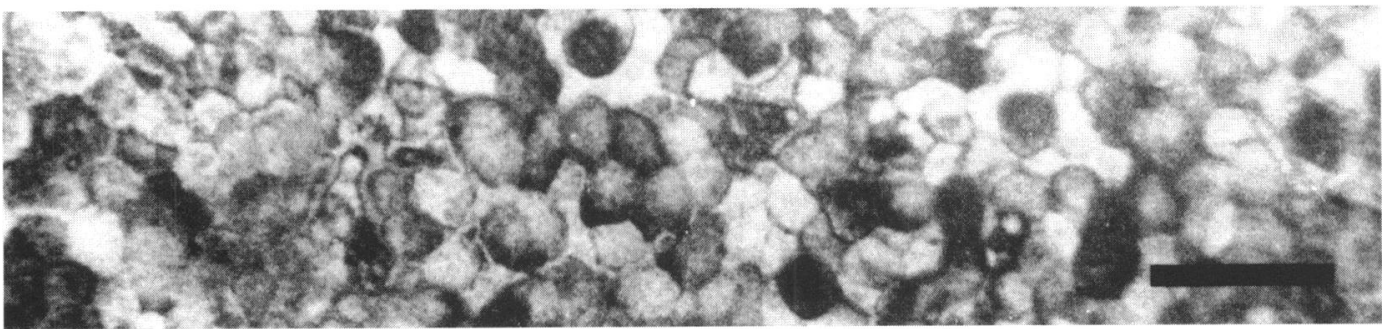

Fig $3 B$

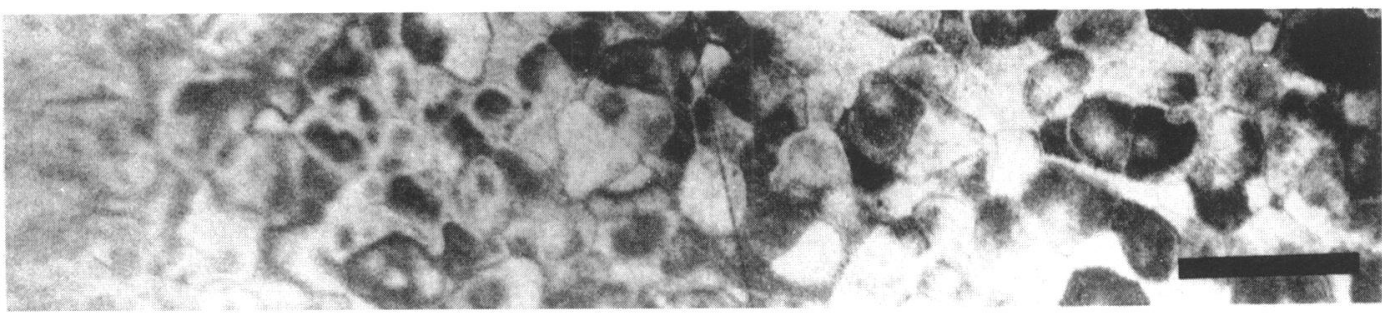

Fig $3 C$

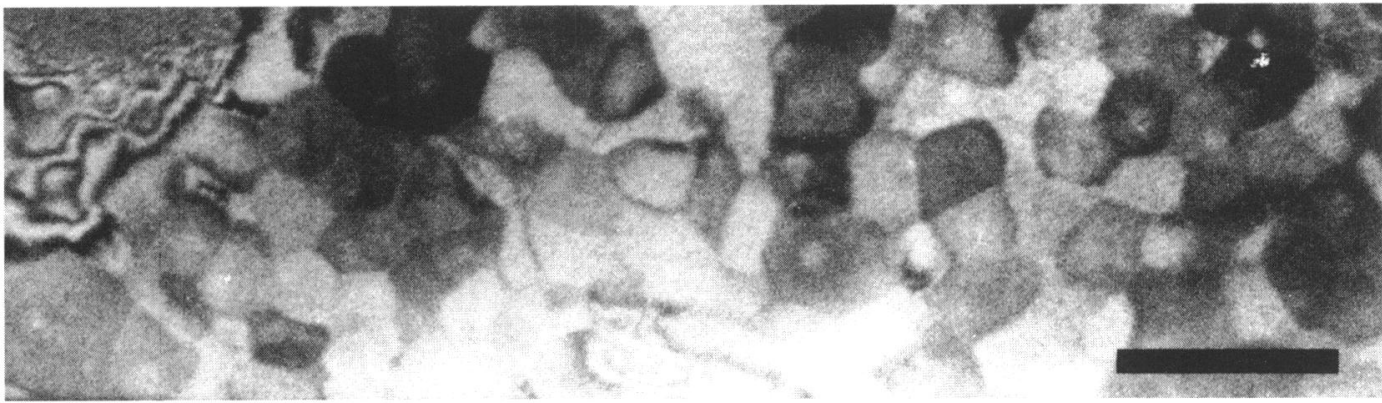

Fig $3 D$

Figure 3 The typical corneal epithelium 6 months after start of extended wear soft contact lens use in the four groups. (A) Group 1, (B) group 2, (C) group 3, (D) group 4. The epithelial cell size is larger in the latter groups.

lowered oxygen level, because rigid gas permeable contact lenses with the same oxygen transmissibility do not change the epithelial cell size. ${ }^{13}$ One possible mechanism is suppression of desquamation. Recently, the concept of apoptosis, or programmed cell death, has been introduced to explain epithelial maintenance. Several factors may trigger apoptosis. In cultured epithelial cells, it is known that exposure to air accelerates differentiation/development,

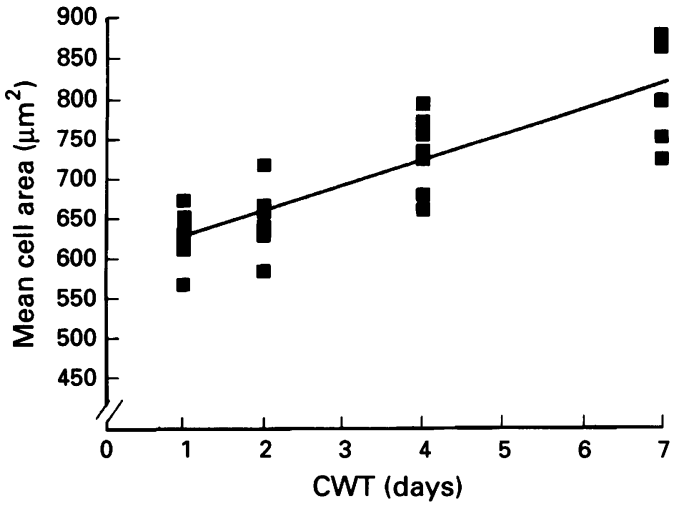

Figure 4 The relation between corneal epithelial cell size and continuous wearing time (CWT). and the same mechanisms may apply to corneal epithelial maintenance. When EWSCLs are worn, the corneal epithelium is not exposed to fresh air, and this may delay programmed cell death. ${ }^{18}$ Since the effect of the extended wear was not apparent at 1 week, but cell size was increasingly larger with overall duration of lens use after 1 month, the epithelium appears to require a long time to stabilise. Further study is necessary regarding the mechanism of enlargement of epithelial cells.

The effect of EWSCL on the corneal epithelium is not an all or nothing phenomenon but is proportional to CWT. Large studies have shown increased rates of corneal pathology with longer CWTs. Although our study was relatively small, it appears that wearing lenses even over a single night increases the risk of corneal complications by altering epithelial morphology.

1 Poggio E, Glynn R, Schein O, Seddon J, Shannon M, Scardino $\mathrm{V}$, et al. The incidence of ulcerative keratitis among users of daily-wear and extended-wear soft contact lenses. $N$ Engl f Med 1989; 321: 779-83.

2 Buehler P, Schein O, Stamler J, Verdier D, Katz J. The increased risk of ulcerative keratitis among disposable soft contact lens users. Arch Ophthalmol 1992; 110: 1555-8. 3 Poggio E, Abelson M. Complications and symptoms in 
disposable extended wear lenses compared with conventional soft daily wear and soft extended wear lenses. tional soft daily wear

4 Dart J, Stepleton F, Minassian D. Contact lenses and other risk factors in microbial keratitis. Lancet 1991; 338: 650-3.

5 Schein O, Glynn R, Poggio E, Seddon J, Kenyon K. The relative risk of ulcerative keratitis among users of dailywear and extended-wear soft contact lenses: a case-control study. N Engl f Med 1989; 321: 773-8.

6 Hamano $\mathrm{H}$, Hori M. Effect of contact lens wear on the mitoses of corneal epithelial cells: a preliminary report. mitoses of corn 9: 133-6.

7 Gilbard J, Gray K, Rossi S. A proposed mechanism for increased tear-film osmolarity in contact lens wearers. $A m$ increased tear-film osmolarity in

8 MacRae SM, Matsuda M, Yee $R$. The effect of long-term hard contact lens wear on the corneal endothelium $C L A O \mathcal{H} 1985$; 11: 322-6.

9 Lemp M, Gold J. The effects of extended-wear hydrophilic contact lenses on the human corneal epithelium. $A m$ Ophthalmol 1986; 101: 274-7.

10 Tsubota $K$, Yamada $M$. Corneal epithelial alterations induced by disposable contact lens wear. Ophthalmology 1992; 99: 1193-6.
11 Tsubota K, Yamada M, Naoi S. Specular microscopic observation of human corneal epithelial abnormalities. observation of human corneal epit

12 Tsubota $\mathrm{K}$, Yamada M, Naoi S. Specular microscopic observation of normal human corneal epithelium. Ophthalmology 1992; 99: 89-94.

13 Tsubota K, Toda I, Fujishima H, Yamada M, Sugawara T, Shimazaki J. Extended-wear soft contact lenses induce corneal epithelial abnormalities. $\mathrm{Br} \mathcal{F}$ Ophthalmol 1994; 78: $907-11$.

14 Tsubota $\mathrm{K}$. Corneal epithelium following intraepikeratophakia. $\mathcal{F}$ Cataract Refract Surg 1991; 17: 460-5.

15 Tsubota K. Chiba K, Shimazaki J. Corneal epithelium in diabetic patients. Cornea 1991; 10: 156-60.

16 Holden BA, Mertz GW. Critical oxygen levels to avoid corneal edema for daily and extended wear contact lenses. corneal edema for daily and extended wear con

17 Tsubota $\mathrm{K}$, Yamada M. The effect of aldose reductase inhibitor on the corneal epithelium. Cornea 1993; 12: 161-2.

18 Regnier M, Schweizer J, Mechel S, Bailly C, Prunieras M. Expression of high molecular weight (67K) keratin in human keratinocytes cultured on dead de-epidermized dermis. Exp Cell Res 1986; 165: 63-72. 